IdeAs

Idées d'Amériques

$11 \mid 2018$

Modernités dans les Amériques : des avant-gardes à aujourd'hui

\title{
Rencontre avec Milton Hatoum
}

\section{François Weigel}

\section{(2) OpenEdition}

Journals

Édition électronique

URL : https://journals.openedition.org/ideas/2366

DOI : $10.4000 /$ ideas.2366

ISSN : 1950-5701

Éditeur

Institut des Amériques

Référence électronique

François Weigel, «Rencontre avec Milton Hatoum », IdeAs [En ligne], 11 | 2018, mis en ligne le 25 juin 2018, consulté le 20 octobre 2022. URL : http://journals.openedition.org/ideas/2366 ; DOI : https:// doi.org/10.4000/ideas.2366

Ce document a été généré automatiquement le 20 octobre 2022

\section{(c) (i) ()}

Creative Commons - Attribution - Pas d'Utilisation Commerciale - Pas de Modification 4.0 International - CC BY-NC-ND 4.0

https://creativecommons.org/licenses/by-nc-nd/4.0/ 


\section{Rencontre avec Milton Hatoum}

\section{François Weigel}

\section{Milton Hatoum}

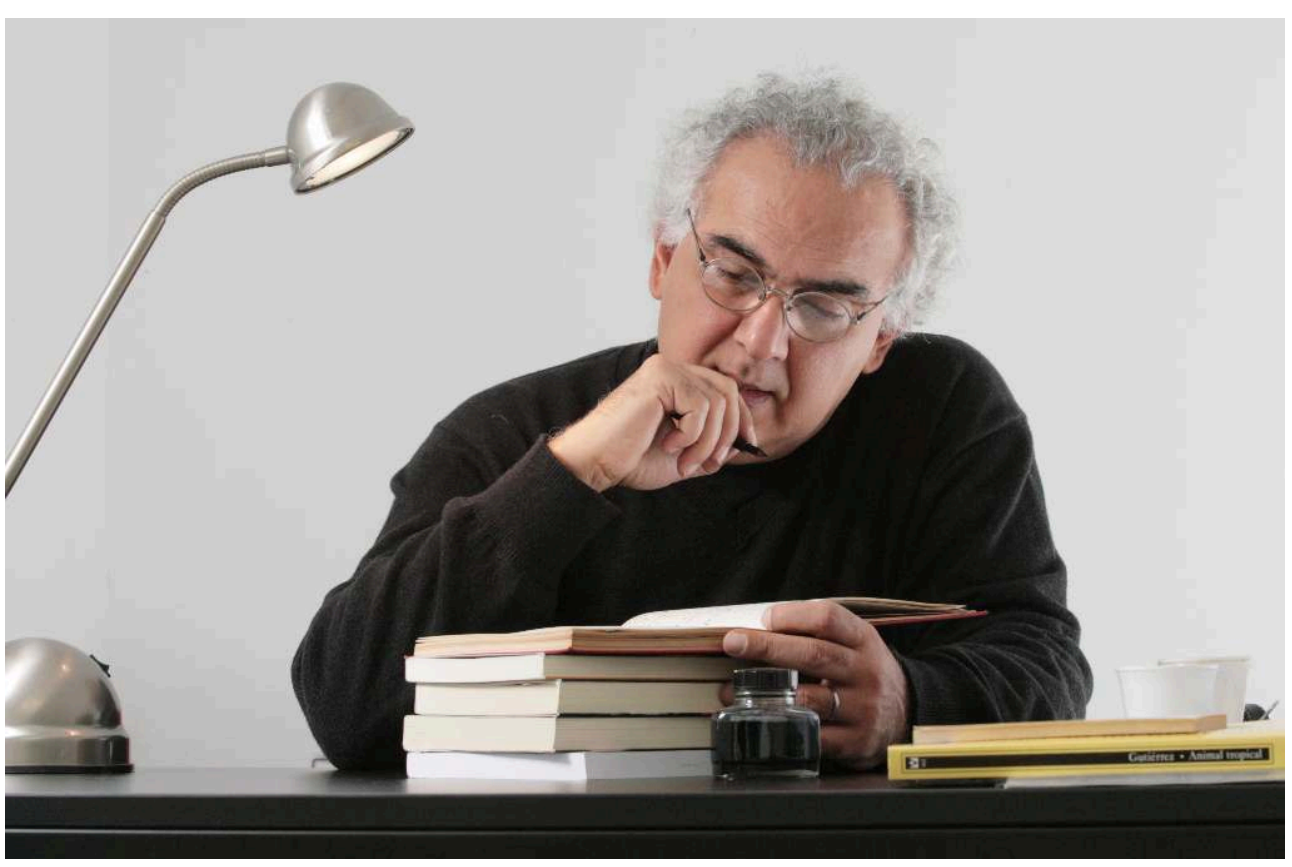

Milton Hatoum

Photographie de Marcos Alves

Depuis plusieurs années, Milton Hatoum est l'un des grands noms de la littérature brésilienne contemporaine. Son œuvre, récompensée par de nombreux prix dans son pays et adaptée au cinéma, à la télévision ou encore en bande dessinée - nous recommandons la très belle transposition du roman Deux frères par les frères Gabriel Bá et Fábio Moon, qui a été éditée en France $^{1}$-, conquiert à la fois la critique universitaire ${ }^{2}$ et un public de plus en plus large. 
3 Né en en 1952 à Manaus, en Amazonie, diplômé d'architecture à l'Université de São Paulo (USP), Milton Hatoum a travaillé comme journaliste culturel et universitaire, d'abord en histoire de l'architecture, puis en littérature. Les questions posées lors de cet entretien l'incitent à évoquer son double parcours intellectuel et professionnel, dans les domaines de l'architecture et de la littérature, ce qui a fait de lui un écrivain particulièrement attentif aux réalités urbaines contemporaines et à la logique de destruction du patrimoine et du tissu social, qui affecte la plupart des villes de son pays. Milton Hatoum, auteur de cinq romans, d'un recueil de nouvelles et d'un livre de chroniques ${ }^{3}$, s'est d'abord essayé à la poésie, sans grand succès, avant que ne soit publié, en 1989, Récit d'un certain Orient (publié au Seuil en 1993)4. Dans ce premier roman comme dans les trois suivants, tous traduits en français ${ }^{5}$, Milton Hatoum, fils d'immigrés libanais, mêle l'imaginaire de l'Orient à la culture amazonienne. La toute dernière fiction publiée par Milton Hatoum, A noite da espera $(2017)^{6}$, ouvre une trilogie romanesque intitulée O lugar mais sombrio, fiction inscrite dans le temps de la dictature militaire et civile (1964-1985) mais aussi dans la capitale Brasilia, ville où l'auteur avait lui-même vécu lors de ses premières années d'études, participant notamment à des manifestations contre le pouvoir politique en place. De Manaus à Brasilia, nous avons demandé à Milton Hatoum d'évoquer la ville littéraire qu'il dessine dans ses fictions. Mais sans plus tarder, promenons-nous dans les rues de villes brésiliennes et surtout dans les venelles enchantées de la littérature, dont Milton Hatoum, grand lecteur tout autant qu'écrivain, parle avec saveur et érudition.

François Weigel : Je vais commencer par une phrase du Récit d'un certain Orient. « Avant de sortir pour aller retrouver Emilie, je pensais encore à toi, qui devais te trouver à Barcelone, quelque part entre la Sagrada Familia et la Méditerranée, assis peut-être sur l'un des bancs de la Plaza del Diamante en train de te figurer ma traversée de l'espace de notre enfance, cette ville imaginaire fondée un matin de $1954 »^{7}$. C'est cette expression, "ville imaginaire ", que je souhaiterais détacher: la Manaus de votre œuvre fictionnelle est une ville construite imaginairement. Est-ce là l'une des forces de la littérature, de pouvoir représenter une ville à travers la mémoire et l'imaginaire?

5 Milton Hatoum : La narratrice, dans cette citation, écrit à son frère qui habite Barcelone. Manaus existe pour elle dans l'imagination, dans un temps passé. L'espace est réinventé par l'imagination, et à travers le temps, le passé. Voilà ce qui m'intéresse : le passage du temps. Beaucoup de lieux dans Manaus sont ainsi revisités par la mémoire. Certains sont encore actuels... Aujourd'hui, il y a une telle aliénation de la population des grandes villes brésiliennes que les Amazoniens ignorent l'existence de certains lieux de Manaus, surtout ceux évoqués dans Cendres d'Amazonie ou Deux frères. Beaucoup de gens ne savent même pas où se trouve, par exemple, l'île de São Vicente, pourtant en plein cœur de Manaus et reliée aux autres quartiers par une sorte de terreplein. Qui plus est, dans le cas du passage cité, la distance temporelle est doublée d'une distance spatiale, entre la narratrice et son frère.

$6 \quad$ F.W. : Ce va-et-vient temporel apparaît dans nombre de vos romans, n'est-ce pas ? Dans Cendres d'Amazonie, plusieurs époques de la ville se superposent aussi...

7 M. H. : Ce jeu temporel est un procédé qui existe depuis un certain temps dans la littérature moderne. Flaubert le maîtrise parfaitement, par exemple. Je pense notamment au «blanc » ou à la césure entre chapitres dans L'Education sentimentale, un procédé que Marcel Proust a mis en lumière [dans un article intitulé « À propos du style de Flaubert ", paru dans la Nouvelle Revue française, en 1920] : Flaubert a admirablement intercalé un blanc, sans transition, entre le retour de voyage de Frédéric et la scène antérieure. Il y a une distance sur la page, en fait un saut de chapitre, qui marque la 
coupure spatio-temporelle. Flaubert signalise cette coupure temporelle, comme l'aurait fait un réalisateur de cinéma. En vérité, Flaubert est très cinématographique, avant la lettre. Le temps et le flux circulent, avec force...

F. W. : Flaubert semble être un écrivain qui vous a marqué et d'ailleurs, dans Récit d'un certain Orient, apparait un perroquet, qui ne manque pas de ramener le lecteur au souvenir d'un autre perroquet, celui de la bonne Félicité, du conte un «Cœur simple $»^{8} .$.

9 M. H. : Un perroquet amazonien, oui ! Flaubert a écrit un conte qui n'est plus tout à fait un conte, mais déjà un roman très court, avec, là encore, de nombreuses ellipses. D'ailleurs, le roman commence par un saut temporel : «Pendant un demi-siècle, les bourgeoises de Pont-l'Évêque envièrent à Mme Aubain sa servante Félicité ». On sait, dès l'incipit, que l'action, au long de 40 pages, va se développer dans l'intervalle d'un demi-siècle. Flaubert a déjà cette intuition romanesque... Jusqu'à cette œuvre folle, Salammbô.

F. W : Pour revenir à votre œuvre, la ville de vos fictions apparaît donc à travers une reconstruction du temps, comme une ville imaginaire, et en même temps il semble que votre formation d'architecte vous pousse à construire cette ville littéraire avec une certaine précision, notamment par le biais de nombreuses indications topographiques. Votre littérature serait-elle donc au croisement du réalisme spatial et d'un travail plus imaginatif, passant par la mémoire?

11 M. H. : C'est une littérature de fond réaliste, sans l'ombre d'un doute. Mais je ne sais pas jusqu'à quel point elle crée une illusion réaliste. La perspective est réaliste, et pour ce qui est de la spatialité, oui, elle a partie liée, je crois, avec ma formation. Peu d'écrivains ont été architectes. Alejo Carpentier a fait des études dans ce domaine, et a écrit sur l'architecture cubaine et La Havane baroque, dans La ciudad de la columnas'. En fait, la pratique de projets architecturaux ne m'a pas seulement servi à penser l'espace romanesque proprement dit, elle m'a surtout aidé à ébaucher l'architecture ou la structure de mes récits - les relations entre personnages, ce jeu temporel, les rencontres et leurs vicissitudes, les voix narratives... J'ai réalisé de nombreux plans sur du papier brouillon, au sujet de cette configuration narrative, qui est en réalité une question très spatiale. J'ai appris à penser l'espace pendant près de cinq ans, à l'Université de São Paulo, et oui, cela m'a beaucoup aidé. Pour la géographie des romans, je réalise aussi des croquis, des ébauches. Et au sujet du monde urbain, le roman est grandement lié à la ville, il a surgi avec la ville, notamment au XVIII siècle, en Angleterre ; il est lié à l'expansion de la bourgeoisie, des industries, de la presse, des grandes villes, Londres principalement, puis Paris...

F. W. : Au Brésil, on peut songer à Rio de Janeiro et à Machado de Assis ${ }^{10} . .$.

M. H. : Exactement, et même à une poignée d'autres écrivains, un peu avant. Mais avec une différence : au Brésil, le temps de Machado, vers la fin du XIX ${ }^{e}$ siècle, n'est pas encore caractérisé par la formation très nette, comme dans la Paris de Balzac ou dans les villes industrielles de Dickens, d'un prolétariat urbain et d'une classe bourgeoise, avec des stratifications sociales clairement différenciées. Le Rio de Janeiro de Machado, c'était une ville où vivaient maîtres et esclaves, avec une classe bourgeoise encore assez restreinte. Cela a ralenti quelque peu l'émergence du roman réaliste au Brésil. Dans cette veine, on trouve certes quelques romans de valeur, comme $O$ cortiço d'Aluísio Azevedo, ${ }^{11}$ mais Rio de Janeiro était encore très différente des villes européennes... Il $n$ 'en demeure pas moins que Machado de Assis, déjà, écrit des romans urbains, bien qu'il 
ne soit, il est vrai, pas encore préoccupé par, disons, une configuration externe, et même s'il est plus enclin à une réflexion ironique sur la société de Rio de Janeiro, ou plus généralement sur la société brésilienne.

F. W. : Je profite de votre commentaire sur Machado de Assis pour évoquer un autre écrivain majeur à la charnière du dix-neuvième et du vingtième siècle, à savoir Euclides da Cunha, auquel vous avez fait allusion dans l'une de vos nouvelles, mais aussi dans une chronique. Parmi les écrits sur la région amazonienne, les essais d'Euclides da Cunha, réunis sous le titre À margem da história ${ }^{12}$, ont une importance significative dans les lettres brésiliennes. Or il me semble que ce titre renvoie à un élément important dans votre œuvre fictionnelle : le fait que Manaus est un espace relativement isolé dans la géographie brésilienne, et peut-être pourrions-nous d'ailleurs établir un parallèle avec la situation de vos narrateurs, eux aussi « à la marge de l'histoire », en tant que fils bâtards, orphelins, individus en mal d'identité, et qui pour cela même seraient aptes à proposer un regard oblique, décentré. Ce parallélisme vous semble-t-il pertinent ?

M. H. : Oui, je crois. Ces narrateurs sont à la marge de leurs familles, de la société. Il y a ce déplacement du narrateur, en effet. Ce ne sont pas des narrateurs faisant partie de l'élite, des classes dominantes. D'ailleurs, à ce sujet, Machado de Assis, j'y reviens une fois de plus, était un génie. Il a travaillé avec ce type de narrateur, des classes dominantes, pour mieux dévoiler leur inconsistance et leur cynisme. Il les dépeint comme des faux libéraux, et dans le fond comme des esclavocrates éhontés.

F. W. : Au juste, qu'est-ce que cela signifie, dans le cas de Manaus, cette idée d'écrire à la marge de l'histoire? Cela implique d'écrire sur des cultures perdues, des familles orientales acculturées, des indigènes réprouvés par la société ? S'agit-il de récupérer un certain passé ? C'est une écriture du « contre »?

17 M. H.: Contre une vision dominante, contre une histoire officielle. Si tu interroges quelqu'un comme Crivela [le maire de Rio de Janeiro, un pasteur évangélique] ou bien quelqu'un comme Alckmin [le gouverneur de l'État de São Paulo, un membre de la très conservatrice Opus Dei], sur le rôle de l'église et du pouvoir politique dans les missions catholiques, ou plus largement chrétiennes, au Brésil, tu peux être sûr qu'ils ne contesteraient aucunement la vision dominante. Pour eux, l'indigène Domingas [un personnage du roman Deux frères] n'est en aucun cas une esclave des temps modernes. Or, pour ma part, tout au contraire, j'ai voulu donner une voix à ceux qui n'ont pas de voix. J'ai fait parler, dans Deux frères, un jeune, fils bâtard de Domingas, qui est une indigène catéchisée, ainsi que l'un des deux frères jumeaux. De même, dans Récit d'un certain Orient, la narratrice est aussi en marge de la famille.

F. W. : Oui, et elle est même internée dans un hôpital psychiatrique.

M. H. : Tout à fait. Mes narrateurs occupent de semblables positions dans Cendres d'Amazonie et Orphelins de l'Eldorado. Dans ce dernier roman, le narrateur est fils d'un homme puissant, mais il dilapide toute la richesse du père. Concernant Euclides da Cunha, j'ai cependant une critique à formuler. Sa marge de l'histoire n'est pas la mienne. Dans ma nouvelle centrée sur Euclides, qui figure dans Um solitário à espreita, il $\mathrm{y}$ a une allusion à Gobineau, ainsi qu'aux principes positivistes qui étaient ceux d'Euclides. Quand ce dernier qualifie l'Amazonie de « désert », il parle d'un désert de la civilisation. Pour lui, les Indiens ne font pas partie de la civilisation. Il ignore la société indigène en tant que représentative d'une communauté humaine. Pour lui, l'indigène n'acquiert une dimension humaine qu'à partir du moment où il s'insère dans l'histoire. C'est une vision qui est encore celle de Vargas Llosa aujourd'hui, pour qui l'indigène du 
Pérou doit cesser d'être indigène pour s'intégrer dans la société péruvienne blanche, en tant que " cholo " [au Pérou, un indigène qui migre vers la ville et s'acculture], selon le terme employé là-bas. Ainsi, quand Euclides da Cunha parle de " marge de l'histoire », il se réfère à ce qu'il considère comme une lacune, et dans le fond préconise d'occuper et de civiliser l'Amazonie. C'est en cela que repose toute l'ambigüité d'Euclides, et les " euclidiens " les plus férus sont très conservateurs, car ils partagent cette vision... J'ajouterais qu'Euclides est un naïf. Un positiviste ingénu. Qui par ailleurs avait des idées socialistes. Machado était bien plus clairvoyant, car Machado était un pessimiste radical, sans espoir.

F. W. : La présence de la nature est un autre élément très présent dans les essais amazoniens d'Euclides da Cunha, d'où ma prochaine question : vous cherchez à fuir les clichés attachés à une représentation pittoresque de l'Amazonie, et ce d'autant que vos romans sont avant tout urbains, mais dans quelle mesure la nature imprègne votre imagination? Je me souviens d'une scène du Récit d'un certain Orient où la narratrice est dans l'avion, de nuit, et décrit les limites imprécises entre la forêt, le fleuve et la ville. La description, par-delà la poésie d'une telle vue aérienne, suggère que la ville rogne de plus en plus la forêt... Ce sont des thématiques sur lesquelles vous mettez l'accent dans votre représentation de Manaus?

21 M. H. : Oui. Cette question est notamment très présente dans Cendres d'Amazonie, avec le personnage de Mundo qui réalise un "Champ de croix», une œuvre artistique écologique et contestataire. Il faut dire que Manaus, au même titre que Belém, est une ville qui fut planifiée dans une certaine harmonie avec la nature, vers la fin du XIX ${ }^{\mathrm{e}}$ siècle, et ce jusque vers les années 1960. Avec le changement impulsé par la Zone Franche, Manaus est désormais en totale disharmonie avec la nature, qui n'est plus intégrée avec elle. Le monde urbain prend ses aises, la destruction de la forêt aux alentours est systématique et rapide.

F. W. : Le fleuve, les pluies... Tous les éléments naturels paraissent parfois, dans vos romans, faire surgir un monde presque merveilleux, au sein même de votre univers réaliste. La description de la nature serait tout près de faire basculer le texte dans une dimension surréelle, irrationnelle. Êtes-vous d'accord sur cette interprétation de la façon dont la nature vient alimenter votre fiction?

M. H. : Oui. Car à Manaus la nature fait partie de la ville. Cela peut sembler exotique pour qui ne connait pas Manaus, mais on y ressent la force de la nature, notamment avec ces pluies torrentielles. La grosse pluie d'hier [à São Paulo], ce n'est rien pour nous, Manauaras. Ces pluies de Manaus ont de quoi effrayer n'importe quel Pauliste! Les rues deviennent alors des rivières, les indices de pluviométrie atteignent des records! La pluie est aussi forte comme image, elle trouble notre vision. Et les averses sont parfois très longues. Je me souviens que quand il pleuvait beaucoup, les gens ne sortaient pas de chez eux pour aller travailler. C'étaient comme des moments solennels en rapport avec la nature, cela changeait le rythme des jours et de la ville. Mais la ville qui s'harmonisait avec la nature, avec ses « igarapés » [ruisseaux et canaux], ses places et ses petits étangs, tout cela a été détruit. Plusieurs places ont été rasées, les igarapés ont été terrassés, la présence de la forêt dans la ville a disparu. Alors que Manaus est au cœur de la forêt, on ne la ressent plus : c'est de la folie, c'est très perturbant... C'est le résultat de l'ignorance et de l'appât du gain. Au Brésil, de façon générale, les administrateurs et les politiques n'ont aucune pensée pour leur ville, ils ne réfléchissent pas. Le seul maire qui a mené une réflexion sur la ville, ici à São Paulo, est 
Fernando Haddad... et il a perdu dès le premier tour des récentes élections municipales de 2016. Simplement parce qu'il est étiqueté PT [Parti des travailleurs, parti de Lula et Dilma Rousseff]. Sous n'importe quelle autre étiquette, il aurait été réélu... Enfin, pour revenir à la littérature, elle est un point de vue critique, plus ou moins subtil, sur la ville. Elle est née dans la ville, mais en même temps elle nous parle de la solitude de l'individu, celui-ci n'ayant déjà plus d'histoire exemplaire à raconter, ayant perdu le sens du romanesque. L'épopée est restée là, dans les limbes du passé. C'est le drame de l'individu dans la vie urbaine. Un drame qui peut d'ailleurs survenir dans d'autres espaces. Diadorim, de Guimarães Rosa, en atteste ${ }^{13}$. L'action de ce roman se situe au cœur du sertão, à l'opposé de la ville, et on y trouverait comme une récupération de l'épopée, ou de traits de l'épopée, et cependant il s'agit bien d'un roman, avec des contrastes forts par rapport à l'épopée. Tout cela est proprement génial...

F. W. : Souvent la Manaus de ces dernières années, contraposée à la Manaus du passé, apparaît comme une ville en ruines, en décomposition, présentant un visage dysphorique, au point que la narratrice du Récit d'un certain Orient, qui revisite sa ville après une longue absence, la "méconnaît », pour reprendre son propre terme. Est-ce là, de fait, un aspect essentiel de votre représentation de la ville?

M. H. : Oui, il y a ici une préoccupation, un regard sur les ruines de la ville. Les ruines de Manaus, qui est une ville si peu connue par les Brésiliens, et qui, je crois, n'avait auparavant jamais été traitée fictionnellement, en tout cas pas avec autant d'insistance. Je ne connais pas de romans, écrits par d'autres écrivains, dont l'intrigue se développe dans Manaus.

F. $\mathbf{W}$ : Des chroniques ou des récits de voyage se sont cependant penchés sur cette ville... mais pas d'autres romans.

M. H. : C'est cela. Et je souhaitais en faire de même pour Brasília dans mon dernier roman. Je dirais, d'une façon générale, qu'il est très important de parler des villes, de travailler ce thème par la fiction. Car les villes d'Amérique latine, et du Brésil en particulier, sont si souvent détruites et reconstruites. Ainsi, du point de vue historique, c'est comme si les villes avaient une vie erratique. Aujourd'hui, aucune capitale régionale du Brésil n'a été préservée. C'est ahurissant. Ici la spéculation urbaine est féroce, les lois ne sont pas assez protectrices et quand elles existent, elles sont contournées allègrement... Sans compter qu'il $\mathrm{y}$ a pour ces questions une grande indifférence, fruit de l'ignorance des Brésiliens, de façon générale, par rapport à leurs villes et à leur histoire. Les Brésiliens, sauf quelques rares et admirables exceptions bien sûr, ne sont pas préoccupés par leur patrimoine. Je l'ai perçu très nettement dans la ville de mon enfance, Manaus, même si bien entendu je connais aussi le sort de bien d'autres villes, Belém, São Paulo, Rio de Janeiro, notamment.

F. W. : Dans certaines interviews, vous avez déclaré « détester » Manaus. C'est une manière de dire ou bien une réalité - que d'une certaine façon vous cherchez à expurger par l'écriture?

M. H. : Cette ville me semble très hostile ! Jusqu'aux années 1960 c'était une belle ville, en harmonie avec la forêt, les petites rivières, la nature. Comme l'a dit Lévi-Strauss, nous avons la nostalgie d'" une époque où existait encore, entre l'homme et la nature, entre les hommes et les espèces naturelles, un certain équilibre ${ }^{14}$. Or cet équilibre a été rompu presque partout. Une journaliste de Manaus m'a envoyé un message pour me demander si j'acceptais d'écrire un billet d'anniversaire en l'honneur de cette ville. Mais je ne vais pas souhaiter joyeux anniversaire à Manaus! Mon billet serait plutôt un 
message de deuil, de consternation totale. L'esprit chauvin de la journaliste en a pris un coup! Mais voilà... À Manaus, avec l'arrivée de la Zone Franche, la destruction fut brusque et brutale. Cela a choqué ma génération, cette tranche d'âge qui a passé son enfance dans les années 1950 et 1960, et qui a connu une autre ville, si différente. En réalité, on n'a aucune considération pour la dignité des habitants. Il n'y a même plus d'ombre à Manaus... Y a-t-il une chose plus absurde, alors qu'on parle d'une ville dont le climat est - encore plus que tropical - équatorial, c'est-à-dire extrêmement chaud et humide ? Pas d'ombre, pas d'arbres, pas de trottoirs : ce n'est pas possible! Et puis $70 \%$ de la population n'a pas accès au réseau d'égouts. Joyeux anniversaire, mais pourquoi ? Je voudrais une ville plus digne, plus humaine. Et ce que je dis renvoie à un autre héritage de mes années à la faculté d'architecture : le fait d'associer l'espace de la ville, la construction de la ville, au monde social et politique. Ces deux choses vont de pair. Quand on parle de la destruction de la mémoire urbaine, on parle, en même temps, des manigances du pouvoir politique et économique. J'ai perçu très clairement, lors de ces années universitaires, que les interventions de l'architecte et de l'urbaniste dépendent du pouvoir politique. L'architecte aura beau rêver, se bercer d'idéaux en concevant de magnifiques projets d'habitation populaire, tout cela sera vain et dérisoire s'il n'a pas d'appuis politiques.

F. W.: Vous parlez de manigances du pouvoir ou des effets dévastateurs de la Zone Franche, et cela nous renvoie tout droit à l'époque de la dictature militaire et civile, que l'on retrouve comme un enjeu central de votre tout dernier roman et qui dans vos romans antérieurs apparaissait déjà en arrière-plan. Comment traiter de ces questions historiques nationales à travers l'écriture fictionnelle?

M. H. : J'ai vécu entre 12 et 30 ans sous une dictature militaire et civile, mais cette époque trouble n'est pas directement évoquée dans mon premier roman, ni dans Orphelins de l'Eldorado, tandis qu'elle apparaît comme toile de fond dans Cendres d 'Amazonie et Deux frères. Hannah Arendt faisait remarquer que le fait d'associer la liberté à la préservation de l'humanité ne met pas fin à l'opposition entre la liberté et la vie, celle-ci structurant tout le champ du politique et demeurant le critère de toutes les vertus spécifiquement politiques. Dans le roman A noite da espera, j'ai essayé d'évoquer cette opposition entre la liberté baillônnée et le développement de la vie en une période de répression violente, de censure et d'iniquités. Le conflit moral et sentimental du narrateur est pour lui plus important que l'engagement politique. La disparition de sa mère lui fait éprouver toute la dureté de ces temps où chacun luttait pour sa survie ou bien se démenait pour trouver de quoi donner un sens à l'existence. $C$ 'est pourquoi la narration circule entre la subjectivité des personnages et les circonstances historiques. Comme dans mes autres romans, j'ai tenté de maintenir un équilibre entre les faits internes et externes, entre l'intériorité des personnages et la réalité, laquelle nous semble parfois hautement improbable.

F. W. : La dictature est donc évoquée de façon très directe dans votre dernier roman, et ce n'est sans doute pas un hasard si le cadre spatial de cette fiction est la capitale du pays. Pourquoi ce choix de placer votre nouveau cycle romanesque dans de nouveaux espaces - Brasília mais aussi l'Europe et la France? Qu'est-ce que ce déplacement spatial vous a apporté, du point de vue thématique et aussi peut-être sur le plan de l'écriture?

M. H. : Mes romans ont un lien avec ma trajectoire personnelle, dans la vie et en tant que lecteur. Brasília, Santos, São Paulo, Madrid, Barcelone, Paris sont des villes où j'ai 
vécu quelque temps, parfois même plusieurs années. Elles font partie de ma formation, de mon passage de l'enfance et l'adolescence vers l'âge adulte. Cela est implicite dans ma trilogie, dans la mesure où j'ai voulu écrire un vaste roman de formation. J'ai en effet cherché à réinvestir ce genre qui s'était développé au XVIII siècle en Allemagne, avant de se consolider lors du siècle suivant, avec le grand roman réaliste français, le roman de la désillusion. C'est un genre universel, inépuisable, très présent dans la littérature contemporaine, ainsi que dans de très grands romans, tels ceux de Gunther Grass, ou comme Origine de Thomas Bernard, ou encore Diadorim de Guimarães Rosa, et tant d'autres... Le déplacement de mon espace romanesque vers Brasília, São Paulo et Paris a partie liée avec ce projet d'écrire un roman de formation. J'y ai donc vécu, mais surtout ces villes apparaissent dans les romans de la trilogie comme des lieux symboliques, où les personnages vivent leurs drames existentiels, moraux et politiques. Je dois d'ailleurs préciser que la genèse de cette trilogie se trouve dans ma nouvelle "Barbara en hiver ", du livre A cidade ilhada (La ville au milieu des eaux, à paraître en Septembre, chez Actes Sud). Dans cette nouvelle, les personnages sont des LatinoAméricains exilés à Paris.

F. W. : Pour ce qui est de la géographie de Manaus, dans vos romans plus centrés sur l'Amazonie, les personnages passent presque toujours par les mêmes endroits. C'est une Manaus particulière, une aire restreinte du point de vue topographique, entre le Bairro dos Educandos, le centre, les quais du fleuve. La Zone franche n'apparaît quasiment pas. Et puis le quartier des immigrés, central dans vos romans, autour de l'église des Remédios (igreja dos Remédios). Avec aussi le marché, Glória, la Cachoeirinha, ces petits quartiers qui faisaient la Manaus d'antan. Ce qui m'amène à une question qui n'est pas strictement littéraire, mais qui n'en a pas moins un rapport avec votre œuvre: comment jugez-vous le poids de la culture syrio-libanaise dans la Manaus d'aujourd'hui ? Cette culture s'est-elle effacée peu à peu ?

M. H. : Cette culture s'est beaucoup diluée, à l'intérieur de la société. Au Brésil, les immigrés se mélangent rapidement. Bien sûr, dans les maisons se préservent des cultures, notamment à travers la nourriture, peut-être la musique également, mais cela aussi se dilue peu à peu, notamment du fait que les enfants de famille arabe se marient avec des Brésiliens d'origines diverses. Ici à São Paulo, il y a encore des clubs libanais et syriens, en particulier des clubs de l'élite. Mais mêmes les membres de ces clubs se sont souvent mariés avec des gens qui n'ont pas ces origines. Mon épouse est petite-fille d'Italiens, et pour eux il en est de même : l'intérêt pour la culture d'origine se perd. Ceci dit, il y a de nombreuses familles d'origine arabe à Manaus, cela est notoire. Et d'une façon ou d'une autre, ils reconnaissent une certaine culture dans mes romans. Pas seulement à Manaus, mais dans le Brésil tout entier, il y a des familles d'origine arabe qui lisent de la sorte mes romans, et en particulier Deux frères, de très loin le plus populaire.

F. W. : Cet hybridisme culturel et ces recherches d'identité portées par vos narrateurs ne viennent-ils pas d'une certaine façon contrebalancer la vision un peu pessimiste - ou simplement réaliste - de la société et de la ville, entraînée dans une logique destructrice ? Il y aurait là, à travers cette récupération d'une mémoire entre l'Orient et l'Amazonie, une certaine espérance, quelque chose d'enchanteur pour le lecteur?

M. H. : Oui, et de ce point de vue le Récit d'un certain Orient est peut-être mon livre le moins amer, en un sens le moins pessimiste. Avec presque une forme de structure carnavalesque, de par la présence très forte de l'immigré, du métissage, ainsi que celle 
du regard étranger, le regard de l'autre. L'étranger n'est pas un intrus, et c'est manifeste dans le cas de ce personnage, l'un des plus intéressants : l'Allemand Dorner, avec sa loupe qui serait comme une métaphore du regard qu'il porte sur l'univers amazonien. Sans que ce regard ne soit cependant idéalisé. Bon, ceci étant, dans Récit d'un certain Orient, le moment principal de l'intrigue est une époque où Manaus n'avait pas encore été affectée par tout ce processus de destruction auquel j'ai fait référence.

F. W. : Pourtant, à la fin du roman, quand la narratrice revient sur les lieux de son enfance, ce thème de la ruine et d'une ville en putréfaction finit par occuper le premier plan.

M. H. : En effet. Comme si ce passage préparait Deux frères et Cendres d'Amazonie. Comme un préambule de ce qui allait ensuite être développé, et qui a lentement mûri dans mon écriture. Mais cela fut totalement intentionnel, le fait de penser et de mettre en consonance la décadence de la famille et la décadence de la ville. Des vies familiales en ruine, ainsi qu'une ville elle aussi en ruine, et en même temps reconstruite à la sauvage... Je pense par exemple à ce bazar indien, la Maison Rochiram, dans Deux frères, totalement chaotique, qui ne ressemble à rien, et en tout cas n'est déjà plus Manaus. Si tu demandes à un Manauara d'identifier des traits de culture française, qui fut si forte à l'époque du cycle du caoutchouc, notamment à travers l'architecture néoclassique et avec l'art nouveau, on ne saura pas te répondre, sauf peut-être pour te donner une information touristique. En tous les cas, le passé n'est plus reconnaissable. Un enfant qui naît à Manaus aujourd'hui va grandir dans une ville difficilement identifiable. Bien sûr, il y a le théâtre Amazonas, l'un ou l'autre monument qui a été préservé, mais cela est si peu...

F. W. : Toujours en lien avec la question de l'espace, surgit une question absolument récurrente du public, dans les événements littéraires auxquels vous êtes conviés, sur d'éventuels rapports entre votre écriture et un certain régionalisme, en lien avec l'identité amazonienne. La critique Tânia Pelegrini a par ailleurs écrit un article dans lequel elle lance, au sujet de votre œuvre, le terme de "régionalisme revisité ». Pourriez-vous donc nous éclairer sur cette question, cette tension qui apparaît dans vos romans entre, d'une part, des éléments locaux, éventuellement "régionalistes » et, d'autre part, non seulement des thématiques universelles mais aussi des éléments thématiques qui renvoient à la pression globalisante, par exemple quand la narratrice du Récit d'un certain Orient se promène près du port et observe l'artificialité d'un quartier envahi par les touristes?

M. H. : Le régionalisme a été important dans la littérature brésilienne. Mais il est embarrassant de considérer des écrivains de l'ampleur de Graciliano Ramos comme " régionalistes ». En vérité, ces auteurs dits régionalistes ont été l'objet de nombreuses attaques dans les années 1930 et 1940, des attaques qui émanaient surtout de Rio de Janeiro. Je pense à un écrivain de Rio qui les détestait, Octávio de Faria, qui était un critique féroce et emblématique de la littérature sociale. Cet auteur, illisible de nos jours, ou en tout cas très peu lu, était pourtant important à l'époque, très présent dans la presse, et s'attaquait au groupe d'écrivains nordestins pour leurs thématiques, prétendant qu'ils étaient incapables d'écrire des romans psychologiques. En réalité, les contempteurs et critiques de l'époque lisaient mal ; ils ne savaient pas comment lire les auteurs du Nordeste, tels Raquel de Queiroz, Jorge Amado, Lins do Rego. Il y a donc eu une méprise, quand on a qualifié cette littérature de « régionaliste ». À dire vrai, même si c'est peut-être un peu moins le cas aujourd'hui, tout ce qui n'est pas produit dans 
l'aire située entre Rio de Janeiro et São Paulo, et surtout ce qui vient du nord-est et du nord, suscite ce regard condescendant, ces critiques négatives. Cependant, le grand roman de notre littérature, de notre langue, se situe dans le sertão, et je parle bien sûr de Diadorim. C'est en tout cas mon avis et, je crois, celui de nombreux critiques. On est loin des grands centres urbains, pour le coup. D'ailleurs, Guimarães Rosa n'en pouvait plus de la littérature urbaine. On a donc façonné cette polarisation. Aux Etats-Unis, quand le terme de "régionalisme » a surgi, il renvoyait, sans aucune connotation péjorative, aux écrivains du fin fond du Sud profond [« Deep South»], qui sont de très grands auteurs. Faulkner, Tennessee Williams, Carson McCullers,... Les NordAméricains classifient tout, d'où ce terme de "régionalisme ». Au Brésil, le terme a été employé pour renvoyer à une littérature dans laquelle prévaut le pittoresque, l'exotique, au détriment du drame humain. Mais avec Quinze de Rachel de Queiroz, Suor de Jorge Amado, et plus tard São Bernardo de Graciliano Ramos [Hatoum cite ici trois auteurs majeurs du mouvement dit «régionaliste»] il n'est plus question de pittoresque, mais bien de drame humain, et c'est évidemment pour cela que ces auteurs ont été traduits. Le pittoresque n'est plus lu au bout de quelques années. Est-ce que tu connais par hasard Paulo Jacob [un écrivain amazonien]? Personne ne le connait aujourd'hui! Il écrivait des romans amazoniens, sans relief du point de vue humain.

F. W. : Au sujet de l'exotisme et du pittoresque, il y a dans Récit d'un certain Orient certains passages où vous semblez jouer avec les représentations exotiques, en particulier à travers une allusion aux cartes merveilleuses écrites par un oncle fasciné par l'Amazonie...

M. H. : Tout cela est ironique, oui ! Et bien des lecteurs n'ont pas saisi cette ironie. Ils n'ont pas perçu ce jeu ironique, de même qu'ils n'ont pas détecté un jeu avec la littérature de voyage, avec Borges et le fantastique. Ce sont ces mêmes lecteurs qui parlent de « régionalisme », d'ailleurs !

F. W. : Pour finir, quel est votre regard sur la littérature brésilienne contemporaine? Êtes-vous un lecteur de la littérature d'aujourd'hui ? Quelle est votre position dans ce panorama littéraire contemporain? Est-ce que vous estimez faire partie d'une génération spécifique d'écrivains?

M. H. : Je connais et j'admire l'œuvre de certains contemporains. Par exemple Ana Miranda, qui a étudié avec moi à Brasília alors que nous avions 16-17 ans. Ni elle ni moi ne savions alors que nous allions devenir écrivains. Ou encore Rubens Figueiredo, un excellent écrivain, et j'ai beaucoup aimé Passageiro do fim do dia, qui parle aussi de la pauvreté, de la segmentation spatiale et sociale, de la souffrance entraînée par les transformations de la ville. J'apprécie également Ronaldo Correia de Brito, pour moi un excellent auteur de nouvelles, en particulier dans Facas. Et j'en lis encore bien d'autres, comme Bernardo Carvalho.

F. W. : Pour la plupart d'entre vous - Correia de Brito, Figueiredo, vous-même -, vous avez commencé à écrire, ou du moins à publier, après la dictature militaire...

47 M. H. : Oui. Je crois que nous faisions alors deux choses : lire et échapper à la police. On se préparait, pour ainsi dire. Néanmoins, je pense que nous avons des langages différents. Par ailleurs, il n'est pas possible de tout lire, tout accompagner. Il y a tant de publications. 

ou moins futile, d'une façon plus ou moins relâchée. Car il existe le « je », et il existe le monde; il existe le «je» de Proust, qui ne se confond d'ailleurs pas avec Proust luimême, ou qui ne l'est que partiellement, et il existe le monde qu'il construit à partir de ce «je », ce monde dans lequel s'observe la décadence de l'aristocratie française, une espèce de maniérisme qui marque la fin d'une époque et le surgissement de cette bourgeoisie kitsch. Il y a donc une grande construction d'un univers, et l'écriture dévide tant de choses. Il y a tout dans Proust - l'architecture, le théâtre, la peinture, la littérature même, le cas Dreyfus et donc l'histoire, les salons décadents,... En somme, ce fut un grand chambardement pour le roman. Après le XIX siècle, le genre se transforme. Avec Proust, le roman, plus que jamais et avec davantage de force encore qu'il ne l'était depuis ses origines, est un genre omnivore, qui contient tout... la confession, la réflexion... tout ! Or cette compréhension est importante pour les jeunes. Parce que ma génération a lu le roman français, a lu Stendhal, Balzac, Flaubert, Hugo, Proust. Le roman, c'est cela. Un romancier doit passer par là. Je n'ai pas commencé par la lecture des auteurs nord-américains de nouvelles, ou par les auteurs minimalistes. Ma formation n'a pas commencé avec Bukowski. Elle a commencé avec Flaubert, à Manaus, à l'âge de 13 ans..$^{15} \mathrm{Ou}$ encore, côté brésilien, avec Graciliano Ramos, qui m'a enchanté pendant mon enfance! Il faut s'imaginer le choc que cela représentait... Un enfant, en province, fasciné par Vies arides de Graciliano Ramos. Ou bien encore par Infância, un livre qui n'est pas simple pour un enfant.

F.W. : Merci Milton Hatoum ! Je crois que ces mots sur la fascination exercée par la lecture constituent une belle façon de conclure cet entretien et d'inciter à lire votre fiction, qui, par le dialogue qu'elle noue avec eux, fait vivre ces grands textes de la tradition littéraire, tout en construisant elle-même son propre univers ! 


\section{NOTES}

1. Gabriel Bá et Fábio Moon, Deux frères, traduit par Michel Riaudel, Paris, Urban Comics, 2015.

2. Il n'y a pas lieu ici de recenser à l'exhaustion tous les travaux critiques sur l'œuvre de Milton Hatoum, mais citons tout de même le texte de Mireille Garcia, puisqu'il concerne au premier chef notre Institut, ayant été récompensé par le Prix de thèse 2015 de l'IdA : Mireille Garcia, Milton Hatoum. Identités, territoires et mémoires, Rennes, Presses universitaires de Rennes, 2017.

3. Le recueil de nouvelles écrites par Milton Hatoum s'intitule A cidade ilhada, São Paulo, Companhia das Letras, 2009 (ce livre, traduit par Michel Riaudel, sera publié en septembre chez Actes Sud). Son livre de chroniques, pour l'heure, n'a pas été traduit : Um solitário à espreita, São Paulo, Companhia das Letras, 2013.

4. Milton Hatoum, Récit d'un certain Orient, traduit du portugais par Claude Fages et Gabriel Iaculli, Paris, Le Seuil, 1993 (1989).

5. Milton Hatoum, Deux frères, traduit par Cécile Tricoire, Paris, Actes Sud, 2015 (2000) ; Idem, Cendres d'Amazonie, traduit par Geneviève Leibrich, Paris, Actes Sud, 2008 (2005) ; Idem, Orphelins de l'Eldorado, traduit par Michel Riaudel, Paris, Actes Sud, 2010 (2008).

6. Milton Hatoum, A noite na espera, São Paulo, 2017. Le livre sera prochainement lancé en France, par Actes Sud.

7. Milton Hatoum, Récit d'un certain Orient, op. cit., p. 15.

8. Le célèbre récit de Flaubert a été traduit par Milton Hatoum, in Flaubert, Três contos, textes traduits du français vers le portugais par Milton Hatoum et Samuel Titan Jr, São Paulo, Cosac Naify, 2004.

9. Alejo Carpentier, La cité des colonnes, photographies de Paolo Gasparini, traduction de Julian Garavito, Montreuil, Le Temps des cerises, 2015 (1970).

10. Classique incontournable de la littérature brésilienne, Machado de Assis est notamment l'auteur des Mémoires posthumes de Bras Cubas (traduit par René Chadebec de Lavalade, Paris, Métailié, 2015 [1881]) et de Dom Casmurro ou les yeux de ressac (traduit du portugais par AnneMarie Quint, Paris, Métailié, 2002 [1899]).

11. Aluísio Azevedo est le grand représentant brésilien du naturalisme et son roman $O$ cortiço relève tous les contrastes sociaux du quartier carioca de Botafogo. Aluísio Azevedo, $O$ cortiço, São Paulo, Ateliê Editorial, 2012 (1890).

12. Euclides da Cunha, À margem da história, Rio de Janeiro, ABL, « Coleção Afrânio Peixoto », 2005 (1909).

13. Après les romans de Machado de Assis, Milton Hatoum évoque là une autre chef d'œuvre de la littérature brésilienne: João Guimarães Rosa, Diadorim [titre original : Grande sertão : veredas], traduit par Maryvonne Lapouge-Pettorelli, Paris, Albin Michel, 2006 (1956).

14. Claude Lévi-Strauss, L'autre face de la lune. Écrits sur le Japon, Paris, Seuil, 2011, p. 179.

15. Milton Hatoum a écrit une belle chronique sur cette époque où il a découvert Flaubert: «Manaus, Flaubert et madame Liberalina». (Liberalina était l'épouse du consul de France, à Manaus). 


\section{AUTEUR}

\section{FRANÇOIS WEIGEL}

François Weigel est docteur en littérature brésilienne / littérature contemporaine. Sa thèse a été réalisée à l'Université de l'Etat de Rio de Janeiro (UERJ) et à l'Université Clermont Auvergne (UCA), dans le cadre d'une cotutelle. Bénéficiaire de la bourse du REFEB, du Ministère des Affaires étrangères.francois.weigel@laposte.net 